\title{
Genèse et propagation de la crue de janvier 1995 dans le bassin de la Meuse française
}

Genesis and propagation of the January 1995 flood in the basin of the French Meuse

Ursprung und Ausbreitung des Hochwassers im Januar 1995 im Becken der französischen Maas

Cyrille Tailliez, Jean Abele et Emmanuel Gille

\section{(Q) OpenEdition}

\section{Journals}

Édition électronique

URL : http://journals.openedition.org/rge/4185

DOI : $10.4000 /$ rge.4185

ISSN : 2108-6478

\section{Éditeur}

Association des géographes de l'Est

Édition imprimée

Date de publication : 1 janvier 2000

ISSN : 0035-3213

\section{Référence électronique}

Cyrille Tailliez, Jean Abele et Emmanuel Gille, « Genèse et propagation de la crue de janvier 1995 dans le bassin de la Meuse française », Revue Géographique de l'Est [En ligne], vol. 40 / 1-2 | 2000, mis en ligne le 06 août 2013, consulté le 08 septembre 2020. URL : http://journals.openedition.org/rge/4185 DOI : https://doi.org/10.4000/rge.4185

Ce document a été généré automatiquement le 8 septembre 2020

Tous droits réservés 


\section{Genèse et propagation de la crue de janvier 1995 dans le bassin de la Meuse française}

Genesis and propagation of the January 1995 flood in the basin of the French

Meuse

Ursprung und Ausbreitung des Hochwassers im Januar 1995 im Becken der

französischen Maas

Cyrille Tailliez, Jean Abele et Emmanuel Gille

\section{Introduction}

En janvier 1995, les crues ont affecté, en France, 31 départements, causant plus de 8 milliards de francs de dégâts. Dans le Nord-Est, le bassin de la Meuse, particulièrement dans le département des Ardennes, a été fortement touché. Cette crue, grave à la fois par les niveaux d'inondations atteints et les durées de submersion, est la conséquence d'une succession d'épisodes pluvieux abondants. Mais c'est la combinaison spécifique des ondes de crue du haut bassin de la Meuse et de ses affluents à partir de sa confluence avec la Chiers qui est véritablement à l'origine du caractère exceptionnel de cet événement, sans doute le plus fort du siècle dans la partie aval du bassin.

\section{Le bassin versant de la Meuse française}

2 Le bassin versant de la Meuse française (10 $430 \mathrm{~km} 2)$ présente une forme allongée et étroite dans sa partie amont, avant de s'évaser largement dans sa partie aval, où le fleuve reçoit ses affluents majeurs (Fig. 1). 
Fig. 1 : Carte orohydrographique du bassin supérieur de la Meuse

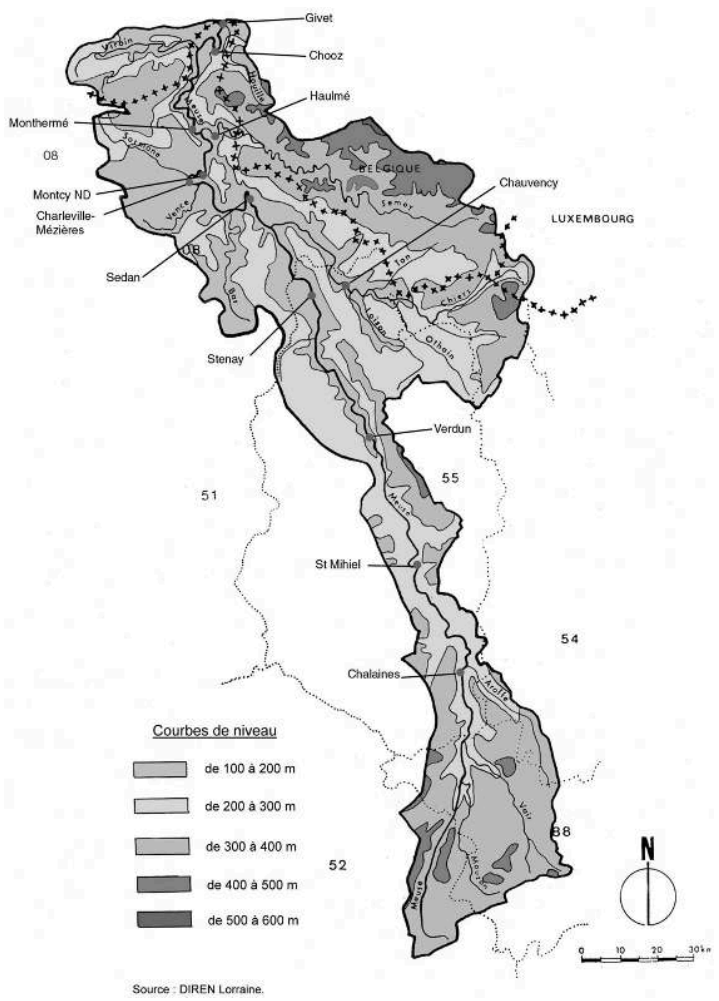

3 La partie amont peut être décomposée en deux sous-ensembles : un haut-bassin en amont de Chalaines, puis un long sillon, conduisant à la confluence de la Meuse et de la chiers.

4 Le haut-bassin de la Meuse représente $1700 \mathrm{~km} 2$, où la Meuse reçoit essentiellement les apports du Mouzon (415 km2) et du Vair (460 km2). Tous ces bassins étant de dimensions proches et relativement réduites, ils réagissent de manière simultanée aux précipitations. Il en résulte la formation d'une onde de crue unique d'amont.

5 La Meuse ne reçoit plus ensuite d'affluent notable, jusqu'à sa confluence avec la Chiers. Sur ce trajet de $230 \mathrm{~km}$, les ondes de crues se propagent en 4 à 5 jours, avec un laminage ou un faible accroissement régulier du débit de pointe de la crue, selon le régime des précipitations au cours de ce transfert. En amont de la Chiers, le bassin versant de la Meuse est de $4145 \mathrm{~km} 2$.

6 À partir de la Chiers et sur une courte distance $(150 \mathrm{~km})$, la Meuse va recevoir un nombre important d'affluents, en descente de l'Ardenne et des contreforts environnants : Chiers (2 $222 \mathrm{~km} 2)$, puis Bar (424 km2), Vence (130 km2), Sormonne (411 km2), Semoy (1 349 km2) (Semoy en France, Semois en Belgique), Viroin (593 km2) et Houille $(234 \mathrm{~km} 2)$. La force de ces affluents, principalement la Chiers et la Semoy, aux courts temps de réaction, peut suffire à générer des ondes de crue marquées sur la Meuse, qui se produisent avant l'arrivée de la crue d'amont et qui peuvent être d'une ampleur supérieure à cette dernière.

7 À cette configuration particulière s'ajoute l'effet du profil en long. Les pentes sont en effet très faibles sur la Meuse et particulièrement sur le tronçon Sedan- Charleville. Dans la partie allongée entre Chalaines et la confluence de la Chiers, la pente est de 0,43 $\%$; elle diminue de moitié $(0,21 \%$ o) entre cette confluence et Montcy-Notre-Dame, 
juste en aval de Charleville. Dans la traversée du massif ardennais, jusqu'à Givet, la pente s'accroît à nouveau $(0,43 \%)$.

Cette faiblesse des pentes s'explique en partie par l'existence de très nombreux méandres qui s'organisent même en un double système de Pagny-sur-Meuse (lieu de l'ancienne confluence avec la Moselle) à Sedan : le cours de la rivière sinue à l'intérieur d'une vallée, elle-même dessinée en méandres de grande amplitude par un ancien cours d'eau de compétence très supérieure.

Les pentes faibles et les méandres qui accroissent les forces de frottement, amenuisent sensiblement les vitesses de transfert des ondes de crue sur la Meuse (à titre de comparaison, les vitesses de propagation sur la Moselle sont environ 1,5 fois plus élevées) et créent des conditions favorables à l'engorgement entre Sedan et Charleville.

\section{Typologie des comportements du bassin de la Meuse française en période de crue}

Les caractéristiques physiques du bassin versant de la Meuse française, esquissées rapidement ci-dessus, expliquent la grande diversité des crues affectant ce bassin, en fonction de la force et la répartition spatiale et temporelle de l'événement pluvieux subi.

Fig. 2 : Bassin supérieur de la Meuse - Comportement-type en réaction à un seul épisode de précipitations uniformément réparties sur le bassin

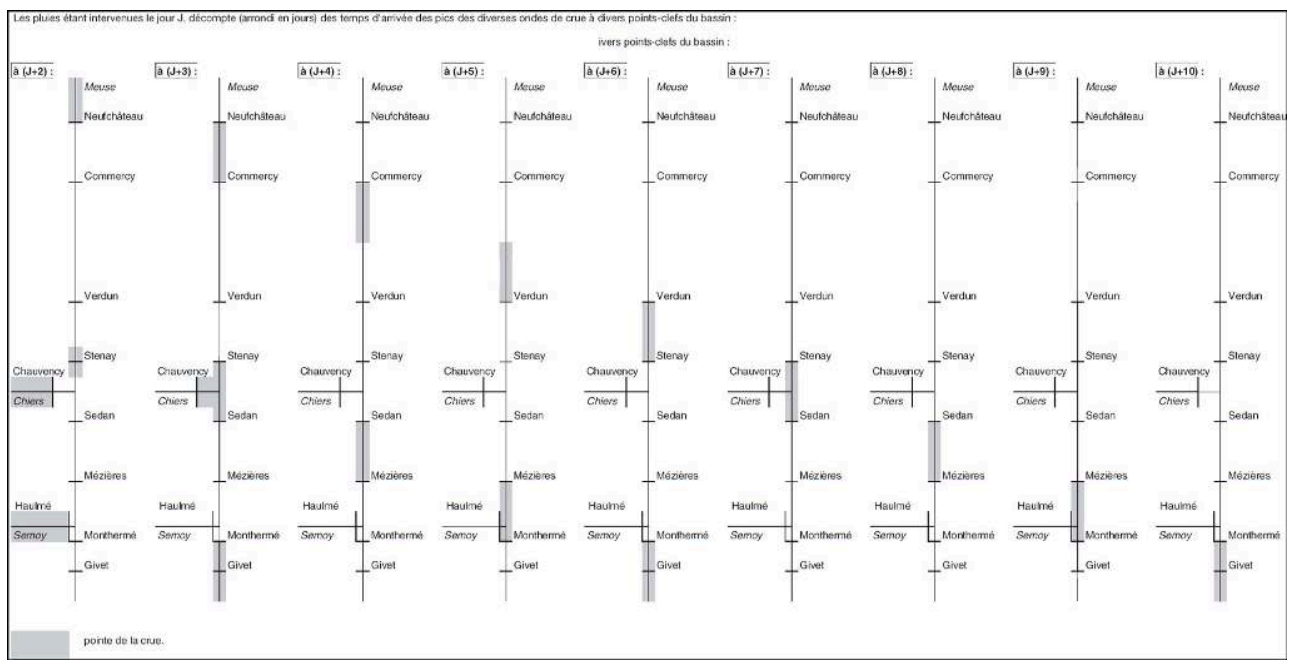

\section{Le cas de l'averse unitaire}

11 Un événement de pluie concentré dans le temps, fort et généralisé à l'ensemble du bassin, génère des réactions simultanées sur le haut-bassin de la Meuse, une crue locale sur l'aval du bassin amont (secteur de Stenay), et des montées sur l'ensemble des affluents ardennais (Chiers, Semoy et Viroin pour les principaux). Un observateur placé au point le plus aval du bassin (Givet) va alors voir se succéder trois trains d'ondes indépendants : en premier lieu, la montée résultant de la crue de la Semoy, qui se fait ressentir à partir de Monthermé (montée renforcée sur Givet de celle du Viroin, ce qui conduit souvent à y observer un maximum anticipé sur celui à celui qui se propage 
depuis Monthermé) ; en second lieu, la montée résultant de la composition de l'onde de la Chiers et de la réaction sur le secteur de Stenay, qui se rencontrent en amont de Sedan; puis, en dernier lieu, l'onde d'amont d'ampleur moindre, qui descend tout le cours de la Meuse (Fig. 1). Pour ce type d'événement pluvieux « unitaire », constaté par exemple lors des précipitations du 27 décembre 1994, ou du 10 janvier 1995, les concomitances sont donc inexistantes.

\section{Cas de succession d'épisodes pluvieux}

Avec un événement pluviométrique prolongé qui se compose d'une succession d'épisodes pluvieux importants, des phénomènes plus nombreux de compositions d'ondes peuvent se développer:

dans le cas, par exemple, de 2 épisodes de pluies importants espacés de l'ordre de 3 jours (Fig. 3A), la crue de la Chiers peut être renforcée par de nouvelles montées de la Semoy et du Viroin ;

avec 3 épisodes de pluies sur une durée de l'ordre de 7 jours (Fig. 3B), c'est la première onde de la crue d'amont qui peut se trouver successivement renforcée par la seconde onde de la Chiers, puis la troisième onde de la Semoy.

Fig. 3 : Bassin supérieur de la Meuse - Exemples de composition d'ondes de crue

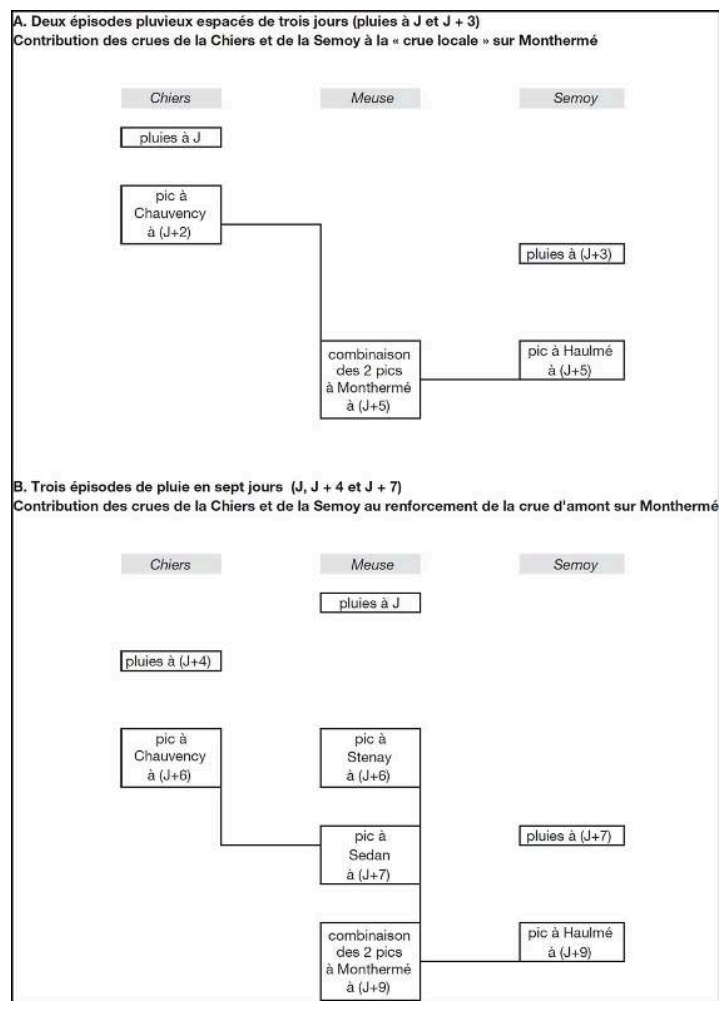

De plus, dans ces situations comprenant une succession rapprochée de forts épisodes pluvieux, deux phénomènes peuvent aussi jouer dans le sens de l'aggravation de la crue : 
Fig. 4 : Formation et propagation de la crue de la Meuse en janvier 1995

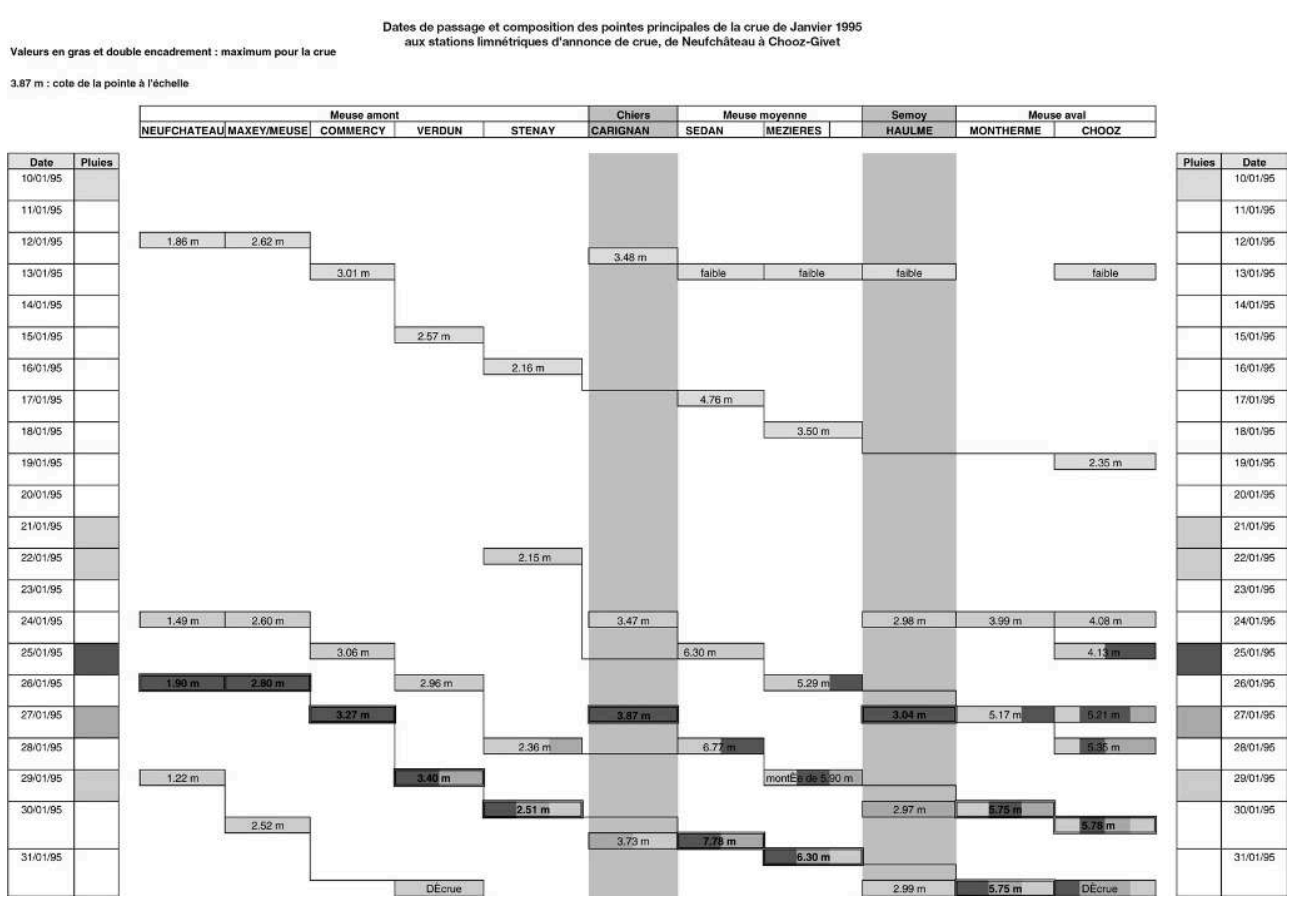
abondantes, surtout sur les parties médiane et aval du bassin $(128 \mathrm{~mm}$ en décembre à Stenay). Les pluies du 27 décembre 1994 (50 mm à Stenay, $91 \mathrm{~mm}$ à La Chapelle, près de Sedan, marge sud du Massif Ardennais) déclenchent une première série de montées des eaux, sans conséquences.

Ensuite, les précipitations, bien que faibles (sauf le 10 janvier 1995, 16 mm à Stenay : voir point 4, 1re phase de la crue), sont quasiment quotidiennes, jusqu'à l'épisode majeur qui commence le 21 janvier. Après celles de décembre, elles tendent à entretenir ou à aggraver la saturation hydrique du sol (de début décembre au 20 janvier, ce sont, par exemple, $179 \mathrm{~mm}$ qui sont tombés à Stenay). 
21 À partir du 21 janvier, quatre perturbations actives, composées de masses d'air très contrastées thermiquement, traversent successivement le bassin de la Meuse :

Le passage d'un premier front chaud, les 21 et 22 janvier, donne de fortes précipitations sur l'ensemble du bassin. Les cumuls de pluies enregistrés sur ces deux jours iront de 40 $\mathrm{mm}$ sur la partie amont du bassin pour atteindre 80 à $90 \mathrm{~mm}$ en plusieurs endroits, notamment sur la partie médiane du bassin, du nord de Verdun à la Semoy. Ces fortes précipitations amorcent une nouvelle montée sur l'ensemble du bassin à partir du 22 janvier.

23 S'il pleut relativement peu le 23, les fortes précipitations reviennent le 24 et surtout le 25 , avec le passage d'une deuxième perturbation, dont le dôme pluviométrique (de 50 à $90 \mathrm{~mm}$ ) est centré sur la Semoy médiane. Ces pluies sont à l'origine de nouvelles montées sur la Meuse et sur ses affluents.

列 29 janvier et donnent respectivement des précipitations de l'ordre de $30 \mathrm{~mm}$ et $40 \mathrm{~mm}$, qui aggravent la montée du niveau des eaux de la Meuse aval.

$\mathrm{Au}$ total, les précipitations sont particulièrement conséquentes en terme de cumul : 209 mm sont tombés à Stenay du 21 au 29 janvier $(267 \mathrm{~mm}$ à Monthermé, à la confluence de la Meuse et de la Semoy), $260 \mathrm{~mm}$ pour tout le mois de janvier et $388 \mathrm{~mm}$ pour les mois de décembre et janvier (599 $\mathrm{mm}$ à Monthermé).

Le tableau 1 montre des temps de retour peu exceptionnels pour des pluies journalières, en général largement inférieurs à 10 ans. Au contraire, le montant mensuel atteint en janvier 1995 à Charleville-Mézières $(297,2 \mathrm{~mm}$ ) affiche un temps de retour de 300 ans (série 1880-1995 des précipitations mensuelles maxima par an, loi Log Normale).

Tableau 1 : Récapitulatif des précipitations journalières ( $\mathrm{P}$ en $\mathrm{mm}$ ) aux stations principales et évaluation des temps de retour ( $T$ en années) correspondants

\begin{tabular}{|c|c|c|c|c|c|c|c|c|c|c|c|c|c|c|c|}
\hline & \multicolumn{2}{|c|}{$27 / 12 / 94$} & \multicolumn{2}{|c|}{$10 / 01 / 95$} & \multicolumn{2}{|c|}{$21 / 01 / 95$} & \multicolumn{2}{|c|}{$22 / 01 / 95$} & \multicolumn{2}{|c|}{$25 / 01 / 95$} & \multicolumn{2}{|c|}{$27 / 01 / 95$} & \multicolumn{2}{|c|}{ 29/01/95 } & $\begin{array}{c}26 / 12 / 94 \\
\text { au } 29 / 1 / 95\end{array}$ \\
\hline & $\begin{array}{c}P \\
(\mathrm{~mm})\end{array}$ & $\begin{array}{c}{ }^{\top} \\
\text { (ans) }\end{array}$ & $\begin{array}{c}P \\
(\mathrm{~mm})\end{array}$ & $\begin{array}{c}\mathrm{T} \\
\text { (ans) }\end{array}$ & $\underset{(\mathrm{mm})}{\mathrm{P}}$ & $\begin{array}{c}{ }^{\top} \\
\text { (ans) }\end{array}$ & $\underset{(\mathrm{mm})}{\mathrm{P}}$ & $\begin{array}{c}{ }^{\top} \\
\text { (ans) }\end{array}$ & $\underset{(\mathrm{mm})}{\mathrm{P}}$ & $\begin{array}{c}\top \\
\text { (ans) }\end{array}$ & $\underset{(\mathrm{mm})}{\mathrm{P}}$ & $\begin{array}{c}\mathrm{T}^{\mathrm{T}} \\
\text { (ans) }\end{array}$ & $\underset{(\mathrm{mm})}{\mathrm{P}}$ & $\begin{array}{c}\top \\
\text { (ans) }\end{array}$ & $\begin{array}{l}\text { Cumul } \\
(\mathrm{mm})\end{array}$ \\
\hline Neufchâteau & 44,0 & 3 & 28,8 & 1 & 19,1 & 1 & 28,2 & 1 & 21,2 & 1 & 14,5 & I & 14,5 & 1 & 290 \\
\hline Stenay & 50,2 & 10 & 15,8 & 1 & 20,6 & 1 & 58,6 & 30 & 32,2 & 2 & 25,2 & I & 31,6 & 2 & 346 \\
\hline $\begin{array}{l}\text { Longuyon } \\
\text { Villancy }\end{array}$ & 41,0 & 3 & 16,2 & I & 26,8 & I & 50,2 & 7 & 39,0 & 2 & 21,0 & ' & 21,7 & 1 & 330 \\
\hline Le Chesne & 15,3 & 1 & 2,2 & 1 & 18,0 & 1 & 49,0 & 6 & 30,0 & 2 & 21,0 & I & 32,0 & 2 & 255 \\
\hline $\begin{array}{l}\text { Monthermé } \\
\text { les Hauts Buttés }\end{array}$ & 62,4 & 15 & 19,8 & 1 & 30,2 & 1 & 54,0 & 6 & 49,8 & 4 & 39,6 & 2 & 48,0 & 3 & 488 \\
\hline Rocroi & 67,3 & 20 & 18,0 & 1 & 23,5 & I & 36,0 & 1 & 35,5 & I & 37,5 & 1 & 46,0 & 3 & 432 \\
\hline
\end{tabular}

\section{Déroulement de la crue de janvier 1995 dans le bassin de la Meuse française}

27 La crue de janvier 1995 relève des crues complexes polygéniques. Son déroulement peut être décomposé en 5 phases, du 10 au 31 janvier (Fig. 4 et 5), si l'on écarte l'épisode du 27 décembre, sans incidence directe. 
Fig. 5 : Hiétogramme à Stenay et hydrogrammes pour la crue de janvier 1995

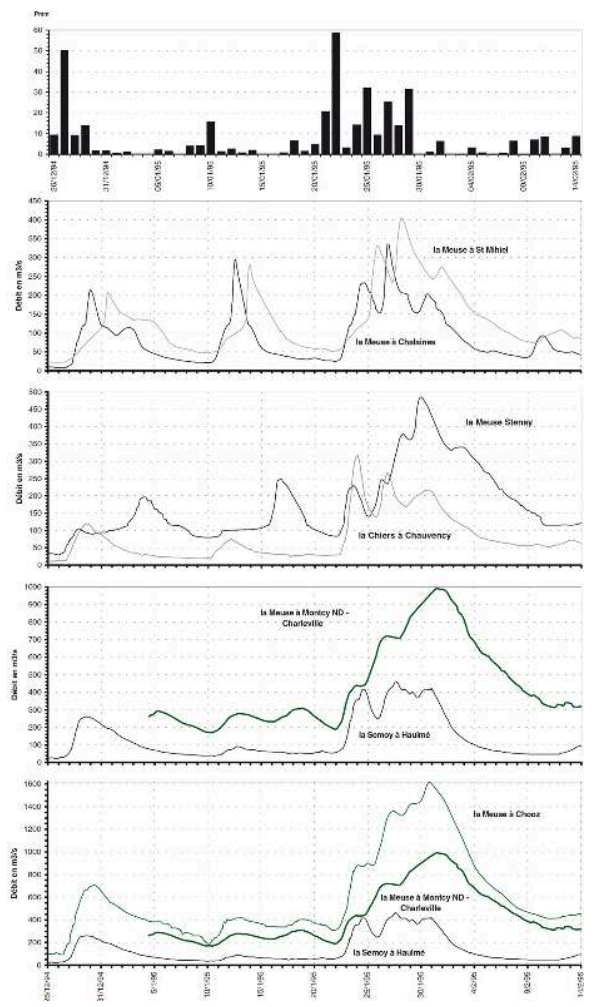

La présentation des différentes phases ci-dessous s'appuie sur les observations faites à Charleville-Mézières / Montcy-Notre-Dame, à l'entrée du massif ardennais et à Givet/ Chooz, à la sortie des Ardennes, frontière franco-belge. Ce secteur a connu les plus forts dégâts.

\section{$1^{\text {re }}$ phase : Crue unitaire du 10 janvier 1995 :}

La 1re journée de pluies significatives, en janvier, a lieu le 10. D’ampleur modérée (et suivies de pluies faibles du 11 au 20 janvier), elles ont pour conséquences une faible montée sur la Chiers, sans effet sur la Meuse, ainsi qu'une onde de crue marquée sur le haut-bassin de la Meuse, qui parcourt, cependant sans renforcement, tout le cours de la Meuse et s'évacue à Givet le 19 janvier. Aucune réaction notable à ces pluies n'est constatée sur la Semoy ou le Viroin.

\section{$2^{\mathrm{e}}$ phase :}

Une pointe locale passe à Montcy le 23 janvier $(437 \mathrm{~m} 3 / \mathrm{s})$, générée par les pluies des 21-22 janvier sur les affluents pré-ardennais (Bar, Vence, Sormone, Vrigne).

- Chooz connaît une forte montée le 23, qui se stabilise par la suite autour de $900 \mathrm{~m} 3$ / s. La première pointe le $24(883 \mathrm{~m} 3 / \mathrm{s})$ est issue des pluies locales des 21-22. Elle est entretenue par l'arrivée de la pointe de Montcy et celle en provenance de la Semoy et donne une 2e pointe, le 25 janvier à $902 \mathrm{~m} 3 / \mathrm{s}$. 


\section{$3^{\mathrm{e}}$ phase :}

- Passage à Montcy le 26 janvier, d'une pointe $(719 \mathrm{m3} / \mathrm{s})$ provoquée, d'une part, par les pluies des 21-22 janvier sur le secteur de Stenay et sur la Chiers (qui connaît son maximum pour la période à $317 \mathrm{~m} 3 /$ ), d'autre part, par les pluies du 25 sur les affluents pré-ardennais (Bar, Vence, Sormone, Vrigne).

- A Chooz, cela se traduit par une pointe de crue le $27(1360 \mathrm{~m} 3 / \mathrm{s})$, résultant de la combinaison de la pointe de Montcy et des pluies du 27 janvier sur l'aval du bassin.

Cette pointe est entretenue jusqu'au 29 (1 $420 \mathrm{~m} 3 / \mathrm{s})$, par la réaction de la Semoy aux pluies du 25. Celle-ci atteint son maximum le 27 à $460 \mathrm{~m} 3 / \mathrm{s}$.

\section{$4^{\mathrm{e}}$ phase :}

Une montée s'effectue à Montcy du 28 janvier au 1er février. Elle se compose ainsi :

- 4a : Les pluies du 25 sur la région de Stenay et sur la Chiers s'associent à celles du 27 sur la Semoy pour former une montée des eaux qui traverse Montcy dans la nuit du 28 au 29 janvier $(830 \mathrm{~m} 3 / \mathrm{s})$.

- 4b : La crue s'aggrave ensuite par le passage d'une combinaison de pointes de crue (988 m3/s, maximum à Montcy), issue des pluies des 21-22 sur la Meuse amont, de celles du 27 dans la région de Stenay et de la Chiers (pas de pointe notable sur ce dernier bassin) et de celles du 29 sur les affluents pré-ardennais.

- 4c : La décrue s'amorce ensuite (encore $976 \mathrm{m3} / \mathrm{s}$ le matin du 1er février à Montcy), ralentie cependant par le passage d'une onde engendrée par les pluies du 25 sur la Meuse amont et celles du 29 sur Stenay et la Chiers.

Au niveau de Chooz, on assiste au passage d'une seule pointe, le maximum de la crue, à $1610 \mathrm{~m} 3 / \mathrm{s}$, le 30 janvier, qui résulte finalement de la composition d'ondes issues de quatre épisodes pluvieux : les pluies du 21-22 sur l'amont, les pluies du 24-25 sur la Chiers et Stenay, les pluies du 27 sur la Semoy et les affluents pré-ardennais, les pluies du 29 sur la partie aval du Massif Ardennais. Les autres pointes se confondent dans la montée de crue ou alors dans la décrue.

\section{$5^{e}$ phase : les ondes dues aux pluies des 27 et 29 janvier sur la Meuse amont}

La 5e phase se perd dans la décrue. Les pluies du 27 et du 29 ont, une dernière fois, fait remonter le haut-bassin de la Meuse, mais sans gravité et, faute d'être alimentées sur l'aval, ces ondes limitées se perdent dans la décrue, à partir de Verdun.

En définitive, sur la Meuse à Charleville-Mézières ou à Givet, l'ampleur de la pointe de crue dépend essentiellement de la composition des ondes de crue. Le poids de la réaction locale immédiate à la pluie, les débits spécifiques des maxima de crue finalement peu élevés, et l'indépendance forte du maximum de Givet par rapport à celui de Charleville, flagrante dans cet exemple de janvier 1995, confirment le rôle déterminant de la structure des épisodes pluvieux. 


\section{Gravité de la crue de janvier 1995 dans le bassin de la Meuse française}

Lors des crues intervenues en France au début de l'année 1995, l'état de catastrophe naturelle a été décrété dans 43 départements.

Dans les Ardennes, les dégâts ont été estimés entre 1,2 et 1,5 milliard de francs, avec 5 000 logements et 1370 entreprises inondés, 11 communes privées d'eau potable, 850 foyers privés d'électricité, 300 de gaz et 3000 de téléphone, 12000 personnes sinistrées, 10125 salariés touchés par le chômage technique. La route nationale 51 et 61 routes départementales ont été coupées, aucune perte humaine n'a cependant été déplorée.

La gravité d'un événement de crue comme celui de janvier 1995 et ses conséquences économiques par les dommages causés, proviennent tout autant des niveaux maxima atteints que de la durée pendant laquelle se sont prolongées les submersions. En termes d'aléas physiques, on peut la caractériser à partir des précipitations, de la durée de submersion, des débits maxima instantanés et des volumes d'écoulement de crue.

\section{A. Les précipitations}

Le premier facteur explicatif de la gravité de la crue subie n'est pas l'intensité de la pluie (tableau 1); c'est, en fait, l'étalement et la répétition de précipitations importantes, sans être extrêmes, sur près de deux semaines. Ceci a eu, en effet, deux types de conséquences : le degré de saturation des sols n'a fait que croître, d'averse en averse (et ce depuis décembre) et les concomitances d'ondes de crue enregistrées dans les Ardennes ont été particulièrement sévères, parce que l'espacement des journées de fortes précipitations a été " en phase » avec les temps de réaction des diverses parties $\mathrm{du}$ bassin. Les pluies se sont donc avérées, sur cet événement, d'une efficacité « optimale ».

46 En tout état de cause, de tels constats sur les pluies et sur la saturation des sols éliminent toutes les hypothèses qui feraient des actions anthropiques sur le bassin la cause principale de la gravité hydrologique de la crue. Il n'en est, bien sûr, pas de même si l'on parle de l'ampleur des dégâts causés aux biens trop inconsidérément implantés en zone inondable.

\section{B. Les submersions}

L'analyse des submersions (tableau 4) menée à l'échelle du barrage de Mézières est représentative de la situation subie dans les Ardennes. Elle fait apparaître une durée de dépassement de la cote d'alerte (correspondant à l'envahissement du lit majeur par les eaux) de près de 25 jours, sans compter le nouveau léger dépassement intervenu après le 13 février. Sur ce délai, la cote de crue grave, qui correspond aux premières atteintes aux biens, a été dépassée pendant 17 jours.

On note, de plus, que l'enchaînement de trois pics de plus en plus sévères, sans que les niveaux redescendent sous la cote de crue grave, a souvent interdit toute intervention de renforcement des mesures de sauvegarde prises en tout début de crue dans les endroits les plus vulnérables, même si les prévisions communiquées par le service 
d'annonce des crues anticipaient correctement l'arrivée des événements successifs, d'ampleur graduelle.

\section{Aspects fréquentiels des hauteurs et des débits maxima instantanés} 1995, en 1784, mais la Meuse n'était pas encore canalisée. En 1926, une crue comparable à celle de 1995 aurait touché Mézières, mais les informations sont lacunaires sur Givet. Quoi qu'il en soit, l'estimation des débits à partir des hauteurs anciennes pose toujours la question de la validité des courbes de tarage et ne garantit jamais l'homogénéité de la série statistique.

51 Aussi, le tableau 2 fournit-il une double estimation fréquentielle des débits maxima instantanés: celle qui s'appuie sur les débits donne un poids relatif fort aux événements de 1993 et 1995 dans une série bien validée mais trop courte pour avoir une réelle signification; celle qui s'appuie sur les hauteurs rend compte de l'état actuel d'appréciation des vieilles observations et comporte une marge d'incertitude certaine, mais reste sans doute plus réaliste.

Tableau 2 : Récapitulatif des pointes maximales de crue (en $\mathrm{m} 3 / \mathrm{s}$ ) (figure 4) et estimation des temps de retour (en années) pour la crue de janvier 1995.

\begin{tabular}{|l|c|c|c|c|c|c|c|c|}
\hline \multicolumn{1}{|c|}{ Cours d'eau } & Chiers & \multicolumn{9}{c|}{ Meuse } & Semoy \\
\hline \multicolumn{1}{|c|}{ Station } & Chauvency & Chalaines & Stenay & Sedan & $\begin{array}{c}\text { Montcy } \\
\text { Charleville }\end{array}$ & Monthermé & Chooz & Haulmé \\
\hline $\begin{array}{l}\text { Débit maximum instantané } \\
\left(\mathrm{m}^{3} / \mathrm{s}\right)\end{array}$ & 320 & 330 & 500 & 860 & 990 & 1310 & 1610 & 460 \\
\hline $\begin{array}{l}\text { Débit spécifique de pointe } \\
\left(1 / \mathrm{s} / \mathrm{km}^{2}\right)\end{array}$ & 188 & 192 & 136 & 132 & 128 & 143 & 159 & 344 \\
\hline Temps de retour (ans) (1) & 50 & 5 & 15 & 60 & 80 & 60 & 70 & 10 \\
\hline $\begin{array}{l}\text { Méthode du maximum } \\
\text { annuel (2) }\end{array}$ & 62 & 5 & 19 & & 41 & & 46 & 16 \\
\hline Méthode du renouvellement (3) & 21 & 3 & 15 & & 45 & & 40 & 14 \\
\hline
\end{tabular}

(1) : temps de retour calculés sur les longues séries d'observations limnimétriques aux échelles d'annonce de crue ; (2) et (3) : temps de retour calculés sur les séries de débits (1965-1997).

D'amont en aval, on peut faire les constatations suivantes :

- la crue sur la Meuse amont est limitée, avec des temps de retour croissants de l'amont vers l'aval, de 5 à 15 ans pour l'onde la plus forte ;

- la Chiers a connu une crue de forte ampleur, avec plusieurs montées élevées ; son pic le plus important a un temps de retour estimé de 50 ans (le 27/01, 3e phase, figure 4).

Il est intéressant de noter que, pour la Chiers, l'estimation du temps de retour par la méthode du renouvellement donne une valeur beaucoup plus faible: cela tient au fonctionnement de ce bassin versant qui n'est pas favorable à l'évacuation des eaux d'inondation (pentes faibles à l'aval) et en conséquence, sans doute à une sousestimation du temps nécessaire à l'indépendance des événements. 

de crue diminué de l'écoulement de base) mobilisés par cette crue aux principaux points-clés du bassin sont élevées sans être exceptionnelles comme en témoignent les coefficients d'écoulement de crue (écoulement de crue / précipitations moyennes du bassin versant) (tableau 3). Cependant, le volume qui s'est écoulé durant le temps où les niveaux étaient supérieurs à la cote de crue grave des services d'annonce de crue, est un indicateur intéressant du volume qu'il serait nécessaire d'écrêter pour limiter les dommages. Ceux-ci représentant plus de $60 \%$ de l'écoulement de crue total à Charleville et Givet, où les montants atteints témoignent de la gravité de la crue (à titre de comparaison, le lac des Vieilles Forges, le plus grand plan d'eau du bassin de la Meuse, fait 5,7 hm3).

Tableau 3 : Récapitulatif des volumes d'écoulement de crue pour l'épisode de janvier 1995 (calculés du 21/01/1995 au 11/02/1995)

\begin{tabular}{|l|c|c|c|c|c|c|}
\hline \multicolumn{1}{|c|}{ Cours d'eau } & Chiers & \multicolumn{3}{c|}{ Meuse } & Semoy \\
\hline \multicolumn{1}{|c|}{ Station } & Chauvency & Chalaines & Stenay & $\begin{array}{c}\text { Montcy } \\
\text { Charleville }\end{array}$ & Chooz & Haulmé \\
\hline Écoulement de crue $\left(\mathrm{hm}^{3}\right)$ & 160 & 141 & 288 & 662 & 1020 & 282 \\
\hline Écoulement de crue $(\mathrm{mm})$ & 94 & 82 & 74 & 86 & 101 & 211 \\
\hline Rang (1966-1997) & 8 & 15 & 14 & 7 & 11 & 6 \\
\hline Volumes précipités (mm) & 216 & 154 & 169 & 198 & 212 & 289 \\
\hline Coefficient d'écoulement de crue (\%) & 43 & 53 & 44 & 43 & 47 & 73 \\
\hline
\end{tabular}

Tableau 4 : Récapitulatif des volumes de crue et des durées de submersion supérieurs à la cote de crue grave, pour la crue de janvier 1995

\begin{tabular}{|l|c|c|c|c|}
\hline \multicolumn{1}{|c|}{ Cours d'eau } & Chiers & \multicolumn{2}{c|}{ Meuse } & Semoy \\
\hline \multicolumn{1}{|c|}{ Station } & Chauvency & $\begin{array}{c}\text { Montcy } \\
\text { Charleville }\end{array}$ & Chooz & Haulmé \\
\hline Cote de crue grave & $1,20 \mathrm{~m}$ & $3,50 \mathrm{~m}$ & $3,40 \mathrm{~m}$ & $2,40 \mathrm{~m}$ \\
\hline Débit correspondant $\left(\mathrm{m}^{3} / \mathrm{s}\right)$ & 95 & 400 & 640 & 200 \\
\hline $\begin{array}{l}\text { Durée de submersion au-dessus de la cote } \\
\text { de crue grave }\end{array}$ & $11 \mathrm{j} 12 \mathrm{~h}$ & $16 \mathrm{j} 12 \mathrm{~h}$ & $14 \mathrm{j} 2 \mathrm{~h}$ & $9 \mathrm{j} 15 \mathrm{~h}$ \\
\hline Volumes au-dessus de la cote de crue grave $\left(\mathrm{hm}^{3}\right)$ & 85 & 426 & 627 & 134 \\
\hline
\end{tabular}




\section{Conclusion}

62 La gravité de la crue de la Meuse de janvier 1995 dans les Ardennes est la conséquence d'une succession de concomitances d'ondes de crue provoquées par une longue période de précipitations soutenues et rapprochées. Si l'ampleur de la crue sur l'ensemble des sous-bassins contributeurs n'a été que relativement forte (à l'exception de celui de la Chiers), elle a pris, sur la Meuse dans les Ardennes (à partir de Sedan), une tournure franchement catastrophique.

Cependant, en terme d'aménagement, pour limiter la vulnérabilité du tronçon de vallée allant de Sedan à Givet, les travaux de l'EPAMA (Etablissement Public d'Aménagement de la Meuse et de ses Affluents) devraient déboucher sur des propositions concrètes visant à écrêter les pointes de crue.

Le rôle évident de l'organisation des épisodes pluvieux dans la composition des crues sur la Meuse aval devrait orienter maintenant les études d'hydrologie de crue de la Meuse en direction de la recherche de probabilité d'occurrence de telles successions pluvieuses.

Il reste qu'il est impossible de dire si l'aggravation constatée au cours des vingt dernières années est une tendance ou un phénomène conjoncturel... La "Meuse endormeuse » sait se faire oublier comme elle l'a déjà fait durant de longues périodes dans le passé.

\section{BIBLIOGRAPHIE}

Actes des journées hydrologiques de Strasbourg. 1987. Crues et inondations. Genèse, méthodes d'étude, impact et préventions. 16-18 octobre 1986. Comité National Français de Géographie. Commission d'Hydrologie Continentale.

Actes du Congrès de la Société Hydrotechnique de France. 1994. Crues et inondations. 23es Journées de l'hydraulique. Nîmes. 14-15-16 septembre 1994. 2 tomes.

Archives de la DIREN Lorraine, service hydrométrique et d'annonce des crues du bassin français de la Meuse.

Archives du SETHY, service hydrométrique et d'annonce des crues du bassin belge de la Meuse.

BCEOM. 1995. Étude fréquentielle des crues de la Meuse entre Neufchateau et la frontière franco-belge, de la Chiers en aval de Chauvency-le-Château et de la Semoy en aval de Hautes-Rivières.

BCEOM Ile de France. 1997. Plan de Prévention du Risque Inondation de la Meuse dans le département des Ardennes entre les Ayvelles et Givet. Définition du zonage.

DIREN Lorraine. AREA. CEGUM. 1994. Etude hydrologique des crues du bassin français de la Moselle, sur la période 1970-1991.

Données pluviométriques fournies par Météo-France. 
Gille E. (1993). - Les crues des bassins de la Moselle et de la Sarre de 1970 à 1991. CEGUM. L'Eau, la Terre et les Hommes. Hommage à René Frécaut. Presses universitaires de Nancy. p. 273-280.

Llamas J. (1993). - Hydrologie générale. Principes et inondations. Gaëtan Morin Editeur. 527 p.

Larras J. (1972). - Prévision et prédétermination des étiages et des crues. Collection du BCEOM. Eyrolles. Paris. 159 p.

Miquel J. (1984). - Guide pratique d'estimation des probabilités de crue. Éditions Eyrolles. Paris. 160 p.

Mosella (1985). - Les crues de 1983 en Alsace et en Lorraine. Revue du Centre d'Etudes Géographiques de l'Université de Metz. Tome XV.

Puginier S. (1995). - Étude des caractéristiques météorologiques associées aux principales crues observées dans le bassin de la Meuse. DIREN Lorraine - Météo France. 1995.

Tailliez C., Warin F. (1992). - Analyse des crues de la Meurthe, de la Moselle et de la Sarre de février 1970 à mai 1983. Mémoire de licence. CEGUM. Université de Metz.

Vidal de La Blache J. (1908). - Étude sur la vallée lorraine de la Meuse. Thèse de Doctorat. Paris.

Vereerstraeten J. (1971). - Le bassin de la Meuse. Étude de géographie hydrologique. Société Royale Belge de Géographie. 339 p.

Warin F. (1993). - L'écoulement de crue des bassins versants de la Moselle et de la Sarre de 1970 à 1991. Mémoire de maîtrise. CEGUM. Université de Metz.

\section{RÉSUMÉS}

L'analyse des circonstances et du déroulement de la crue de janvier 1995 dans le bassin de la Meuse française met en évidence les conséquences catastrophiques sur l'aval du bassin, qui peuvent résulter d'un événement pluvieux soutenu et de longue durée, alors que la crue est restée d'ampleur limitée dans le haut-bassin comme sur une grande part des affluents. Il en ressort notamment le rôle des précipitations prolongées sur la saturation hydrique des sols et sur la composition des ondes de crues issues de sous-bassins aux temps de réponses contrastés.

Analysis of the circumstances and progress of the flood of January 1995 in the French Meuse basin reveals the catastrophic consequences on the downstream areas of this basin, which can result from a sustained rainfall events of long duration, while the flood, in most of the tributaries of the upper basin, remained of limited size. It puts notably the role of prolonged rainfalls on hydrological saturation of soils and on the composition of flood waves issuing from sub-basins at contrasting response times.

Die Analyse der Umstände und des Ablaufs des Hochwassers im Januar 1995 im Becken der französischen Maas macht die katastrophalen Auswirkungen flussabwärts im Becken offenkundig, die durch einen starken und langdauernder Regenfall entstehen können, während das Hochwasser im oberen Becken und in einen grossen Teil der Zuflüsse von bescheidenen Umfang blied. Dabei ergibt sich besonders die Bedeutung verlängerter Niederschläge für die hydrische Sättigung der Böden und für die Zusammensetzung der Hochwasserwellen aus den Beckenteilen zu unterschiedlichen Zeiten. 
INDEX

Keywords : 1995, catchment area, flood, inondations, Meuse

Mots-clés : 1995, bassin versant, crue, inondations, Meuse

Schlüsselwörter : 1995, Abflussbecken, Hochwasser, Maas, Überschwemmungen

\section{AUTEURS}

\section{CYRILLE TAILLIEZ}

Centre d'Études Géographiques de l'Université de Metz - UFR Sciences Humaines et Arts - Ile du Saulcy, 57045 Metz Cedex 01

\section{JEAN ABELE}

Ministère de l'Équipement, des Transports et du Logement - Direction de la Recherche et des Affaires Scientifiques et Techniques - Tour Pascal B 92055 Paris La Défense Cedex 4

\section{EMMANUEL GILLE}

Centre d'Études Géographiques de l'Université de Metz - UFR Sciences Humaines et Arts - Ile du Saulcy, 57045 Metz Cedex 01 